\title{
The Electromagnetic Will
}

\author{
Johnjoe McFadden 10
}

check for updates

Citation: McFadden, J. The Electromagnetic Will. NeuroSci 2021, 2, 291-304. https://doi.org/10.3390/ neurosci2030021

Academic Editor: James Sonne

Received: 24 July 2021

Accepted: 23 August 2021

Published: 29 August 2021

Publisher's Note: MDPI stays neutral with regard to jurisdictional claims in published maps and institutional affiliations.

Copyright: (C) 2021 by the author. Licensee MDPI, Basel, Switzerland. This article is an open access article distributed under the terms and conditions of the Creative Commons Attribution (CC BY) license (https:/ / creativecommons.org/licenses/by/ $4.0 /)$.
Faculty of Health and Medical Sciences, University of Surrey, Guildford GU27XH, UK; j.mcfadden@surrey.ac.uk; Tel.: +44-1483-686-494

\begin{abstract}
The conscious electromagnetic information (cemi) field theory proposes that the seat of consciousness is the brain's electromagnetic (EM) field that integrates information from trillions of firing neurons. What we call free will is its output. The cemi theory also proposes that the brain has two streams. Most actions are initiated by the first non-conscious stream that is composed of neurons that are insulated from EM field influences. These non-conscious involuntary actions are thereby invisible to our EM field-located thoughts. The theory also proposes that voluntary actions are driven by neurons that receive EM field inputs and are thereby visible to our EM field-located thoughts. I review the extensive evidence for EM field/ephaptic coupling between neurons and the increasing evidence that EM fields in the brain are a cause of behaviour. I conclude by arguing that though this EM field-driven will is not free, in the sense of being acausal, it nevertheless corresponds to the very real experience of our conscious mind being in control of our voluntary actions. Will is not an illusion. It is our experience of control by our EM field-located mind. It is an immaterial, yet physical, will.
\end{abstract}

Keywords: consciousness; electromagnetic; neuron; will; determinism; ephaptic; field; computing

\section{Introduction to the Cemi Field Theory}

When a neuron fires, the motion of matter particles, ions, through ion channels in and out of neuronal membranes generates the action potential that travels down the length of the neuron until it reaches the synapse where it triggers the release of chemical neurotransmitters that transmit signals to downstream neurons. Although the details are far from clear, it is generally assumed that each neuron performs some kind of rule-based information processing on its many inputs to generate its output, somewhat similar in concept, if not in physical realization, to a computer logic gate. Most neurobiologists also accept, in broad terms, the computational theory of mind that proposes that networks of neurons implement something akin to computer algorithms to process sensory information to generate intelligent motor actions such as speech. The theory that will be discussed here accepts all of this, but it proposes that, alongside the matter-based algorithmic information processing performed along the brain's neuronal wires, a quite different form of computation is implemented through the interactions of EM field with neurons leading to motor actions. These we experience as what we call "will".

The idea that mental states reflect the dynamics of some kind of field goes back at least as far as the Gestalt psychologists who, in the early decades of the twentieth century, insisted that the holistic properties of perception must be instantiated in some kind of field. For example, they pointed out that musical notes get their value by being perceived as part of a whole melody, just as a beat gets its value only as part of a whole rhythm. Similarly, words are perceived in their entirety, not as a collection of letters. One of their founders, Wolfgang Köhler, proposed that these gestalt properties of object perception are encoded in electrochemical "brain-field[s]" that are isomorphic with "the field of a percept" [1]. The idea fell out of fashion when the underlying neuronal basis of brain function was discovered, as there did not seem to be any way of encoding fields within the discrete neuronal substructure of the brain. Nevertheless, the idea of some kind of 
field existing in the brain persisted in the latter half of the twentieth century as a means of accounting for the binding problem of consciousness-how our conscious mind integrates information distributed among billions of spatially separated neurones to generate the unity of conscious experience. This is essentially the same problem with which the gestalt psychologists had been wrestling but now generalized beyond perception to any conscious state.

The philosopher Karl Popper [2] proposed that consciousness was a manifestation of some kind of overarching force field in the brain that could integrate the diverse conscious information held in distributed neurones. The idea was further developed and extended by Popper with Lindahl and Århem [3] and then by the pioneering experimental neurophysiologist, Benjamin Libet $[4,5]$. However, these researchers considered that, although a field, the conscious mind could not be a manifestation of any known form of physical field and so its nature remained mysterious. In the 1990s, the Nobel Prize-winning mathematician Roger Penrose and the anaesthetist Stuart Hameroff proposed their Orch OR theory [6-8], claiming that consciousness is a manifestation of quantum coherence between neuronal microtubules in the brain. Quantum coherence is a field property-a state in which matter particles behave like a unified force field. It has been proposed to be involved in biological phenomena such as photosynthesis, olfaction, enzyme catalysis or avian navigation (for review see in [9]) but, in these cases, is limited to very small systems of single or handfuls of molecules and is generally considered to be infeasible for large systems that decohere (loss of coherence) very rapidly to lose their quantum nature at physiological temperatures. As the Orch OR theory requires billions of molecules to remain coherent across the entire brain for physiologically-relevant timescales, it is generally considered infeasible.

The dominance of the neuron dogma led, in 2009, the influential neurophysiologist Horace Barlow to insist that "there is no evidence for anything immaterial in our heads, or in any other animal" [10]. Yet, despite Barlow's confident assessment, there is abundant evidence for a physical yet immaterial entity in the brain. The existence of an electromagnetic (EM) field in the brain has been known since the nineteenth century and is routinely detected by medical scanning techniques such as EEG and MEG. EM fields are physical fields that are just as real as matter but composed of perturbations of the EM field that fills the entire universe. Electricity and magnetism are complementary manifestations of electromagnetism which, despite being immaterial, clearly has widespread causal effects on matter. It is responsible for pretty much all of chemistry, biology and harnessed in technologies from radio transmission to the modern computer. Given the importance of EM fields in artificial technology, it is hardly surprising that they play a role in the most complex biological technology in the known universe: the human brain.

The sources of the brain's EM field are not entirely clear but certainly include action potentials and synaptic potentials that involve the motion of ions through neuronal membranes. Movement of electrical charge generates an electromagnetic (EM) field perturbation or wave, as described by Maxwell's famous equations. The motion of ions moving in and out of around 86 billion neurons and 125 trillion synapses in the human brain thereby generates the most the most complex EM field in the known universe, and one that is routinely measured in brain scanning techniques such as EEG and MEG. Nevertheless, despite the fact that Maxwell's laws are symmetric-EM fields generated by moving charges influence the motion of moving charges-brain EM fields were generally considered functionally impotent, as irrelevant to brain activity as the steam whistle on a steam train is irrelevant to its locomotion.

I should emphasize that the lack of interest in EM field interactions between neurons was not a consequence of any evidence against EM field interactions in the brain. The absence of evidence was solely due to the difficultly of obtaining experimental evidence of EM field communication. While techniques, such as patch-clamping and neurotransmitter studies, were rich sources of experimental data on electrochemical neurotransmission, there were no similar techniques available to study EM field interactions. 
My own interest in brain EM fields came about when considering the binding problem. It occurred to me that shifting the substrate of consciousness from the matter of the brain to its equally physical but immaterial EM field effortlessly solved the binding problem as fields automatically integrate and thereby bind information encoded in their sources. This is what we mean by a field. For example, your weight at this moment represents an instantaneous integration-performed by the gravitational field of the Earth-of the mass of your body and the mass of the entire planet. Similarly, the EM field at any point in your brain represents an integration and binding of information encoded by every single firing neuron in your brain.

This shift from the matter of the brain to EM fields generated by neuron firing also made sense of the discovery that synchronously-firing neurons are the best established neural correlate of consciousness (NCC) [11,12]. If, as I propose, the seat of consciousness is an EM field, then it will be subject to wave interference. Most pairs of neurons fire asynchronously to generate EM field waves whose peaks and troughs do not align and so will tend to cancel each other out by destructive interference to generate a zero-net field. However, neurons that fire synchronously will generate EM fields that superimpose and strengthen each other by constructive interference. The cemi field theory thereby predicted that the (EM field-encoded) contents of consciousness generated mostly by synchronously-firing neurons would correlate with synchronously firing neurons [13].

Work conducted by Wolf Singer and colleagues in the 1990s demonstrated that neurons in the monkey brain fire synchronously when the animal attends to the stimulus [14], and additional studies confirmed and extended these findings [15]. Many subsequent studies demonstrated that neural synchrony also correlates with conscious perception in humans. For example, neural synchrony patterns were found to correlate with conscious recognition by subjects exposed to optical illusions [16], and conscious auditory perception was shown to be correlated with long-range synchrony of gamma oscillations [17]. As far as I am aware, no theory of consciousness, other than the cemi field theory and the other EM field theories of consciousness, necessarily predict, rather than merely incorporate, the correlation between synchronous firing and consciousness.

I first described the conscious electromagnetic information (cemi) field theory of consciousness in the final chapter of a book published in 2000, writing that 'this em-field ... [of the] brain integrate[s] information from all of the calculations ... performed by ... [its] logic gates' [18]. I provided a more detailed description of the cemi field theory in two papers published in 2002 [13,19]. Remarkably, similar theories were proposed around the same time by neurobiologist Susan Pocket [20,21], the neurophysiologist E. Roy John $[22,23]$ and the neurophysiologists Fingelkurts and Fingelkurts [24,25]. Updates on the cemi field theory were published in 2006 [26], 2013 [27,28] and 2020 [29].

\section{The Electromagnetic Will}

A key aspect of the cemi field theory, which is not shared by all EM field theories of consciousness, is that it provides a physical mechanism for the action of what we call "free will": the initiation of voluntary behaviour. The basic idea is summarized in Figure 1, which shows three hypothetical firing neurons, A, B and C each with several inputs and a single output that will eventually (via several downstream neurons) initiate a motor output. Also represented, as wavy lines in the figure, is the EM field generated by neuron firing. In the top panel A is represented the situation for neurons engaged in non-conscious actions, such as directing the precise motion of lips and tongue to generate words. Each neuron acts independently to generate an output based on information processing of its several inputs. Each neuron's output is independent and none is influenced by the EM field generated by their firing.

In the bottom panel B is represented the situation for neurons engaged in initiating voluntary action such as choosing to say a particular word: the output of consciousness or free will. The situation is exactly the same as A except that the EM field generated by the firing of neurons $\mathrm{A}, \mathrm{B}$ and $\mathrm{C}$ now provides an additional electromagnetic input to the firing 
decision of each neuron, essentially the neuron is acting as an EM receiver, perhaps pushing two towards firing and another away from firing. This input represents an integration of the information encoded in the firing of each neuron. Although only three neurons are represented in the figure, in reality, this input will represent the integration of information encoded by all firing neurons in the brain. This, I propose, is the physical instantiation of what we call free will. Note that, in the cemi field theory, although EM fields make an input into neuronal/synaptic computation: they are never solely responsible for a conscious state or output. Consciousness is how we experience EM field inputs into neuronal computation. The input may be tiny compared to the underpinning neuronal computation, but it may still be sufficient to deliver awareness of initiating a voluntary behaviour.

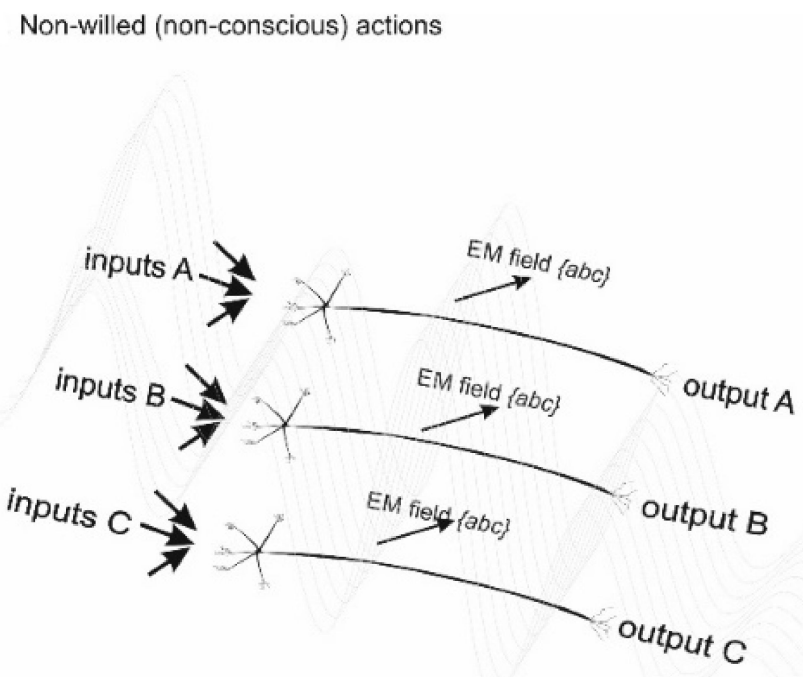

Willed (conscious) actions

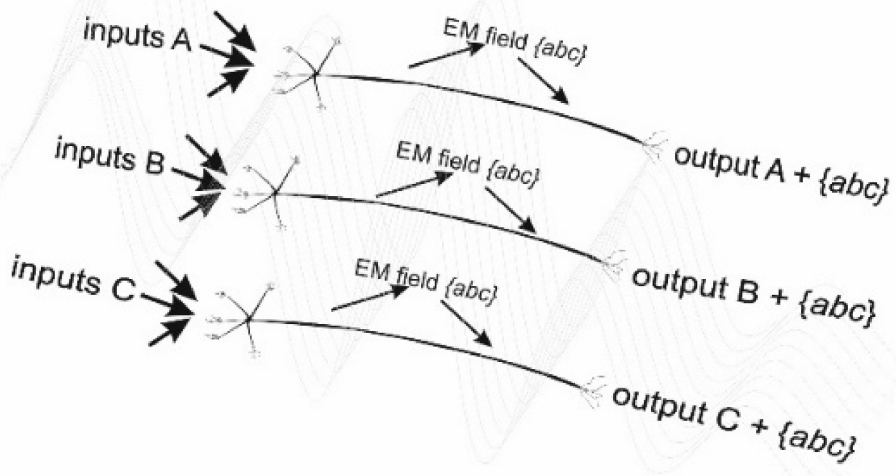

Figure 1. Willed (conscious action) vs. non-willed(non-conscious actions). In both cases, A, B, and C are the inputs and outputs of some neuronal computation by three adjacent non-interacting neurons. What we call free will is then our experience of the input of the EM field generated by all three neurons on the output of all three neurons. In reality, the brain' EM field is a product of the physical integration of the activity of many billions of neurons and free will is then the influence of that EM field-based integration on our actions.

\section{Comparison of Cemi Field Theory with IIT and Global Workspace Theory}

The idea that consciousness represents integrated information in the brain has recently been revived by Giulio Tononi and colleagues [30-37] who formulated the Integrated Information Theory (IIT). The theory proposes that the physical substrate of consciousness 
corresponds to the information encoded within a system that has the highest value of a parameter known as $\Phi$ (phi), which is a measure of the degree of information integration. In particular, Tononi defines integrated information as 'the amount of information generated by a complex of elements, above and beyond the information generated by its parts'. The theory has gathered a lot of support but has also been subject to subject to several criticisms [38,39] which I will not reiterate here but instead compare IIT to cemi field theory.

Although, as far as I am aware, although $\Phi$ has only been applied within IIT, it is essentially a variant of mutual information which, in probability and information theory, is a measure of the mutual dependence between different variables within a system. Measures of mutual information have been evaluated for a wide variety of complex systems, ranging from social networks [40,41] to communities [42,43], ecological networks [44], financial [45] and institutional networks [46]. It is also cornerstone of many systems biology approaches that, for example, examine coherence of physiological systems [47], infer transcriptional regulatory networks from correlated patterns of gene expression patterns [48], describe the hierarchical structure of immune networks [49] and the interdependence of biochemical networks [50]. All of these systems are characterized by high levels of integrated and complex information. For example, with $10^{12}$ cells compared to the $10^{11}$ neurons in the brain and communicating via at least a hundred different cytokines, chemokines and hormones, the immune system processes a vast amount of integrated information and is capable of entering a vast number of distinct states yet does so entirely without awareness. Similarly, high values for mutual information have also been described for artificial intelligence systems, such as in robotics [51], deep neural networks [52] as well as random Boolean networks [53].

In his 2008 IIT 'Provisional Manifesto', Tononi insists that 'to generate consciousness, a physical system must be ... unified; that is, it should be doing so as a single system, one that is not decomposable into a collection of causally independent parts [34]. This is as true for the above systems as it is true for neuronal computation, yet none are considered to possess conscious states.

However, as argued in my recent paper [29], the integration of IIT shares with other socalled systems rich in 'integrated information' the feature that the information integration is temporal in the sense in the sense that it is accomplished through an event that is dependent on a multiplicity of upstream causes. For example, an extraordinarily complex computation performed by a cloud computing operation performed by many computers at different locations on different continents that input vast quantities of interdependent data and mutual information on the climate of the planet may generate the binary code 00101010 that instructs a single printer to print the number 42 . That binary code can be considered to integrate its highly complex inputs, but it has no knowledge of those inputs nor the calculations performed to generate the state of the binary code. The integration is via a causal chain of operations in time, rather than physical integration in space and cannot be equivalent to, say, the state of a conscious mind contemplating the influence of human activity on the current climate. Similarly, the differentiation of a single $\mathrm{T}$ cell in a human may be influenced by many different chemokines and cytokines secreted by other immune cells as well as hormonal signals released by the endocrine system, together with genetic and environmental factors that stretch back to a person's conception or, taking into account, that person's evolutionary history going back to the origin of life nearly four billions years ago. It would be preposterous to claim that any part of this system is conscious on the basis of this kind of causally integrated chain of information processing. IIT predicts that each of these systems is conscious, whereas the cemi field theory insists that, as their information processing is only causally, rather than physically integrated, they are not conscious.

IIT is also refuted by many routine observations. For example, IIT claims that consciousness is associated with the information processing in the brain that has the highest values of $\Phi$. This claim is refuted by our subjective experience whenever complex thoughts, such as those experienced when we are solving a complex problem, are supplanted by a simple neuronal computation, such as those involved in generating the experience of pain 
if we accidently stub our toe. The cemi field theory easily accounts for this phenomenon by predicting that painful stimuli stimulate large (though likely informationally simple) perturbations in the brain's EM field. This prediction is confirmed by many EEG and MEG experiments, for example [54].

The cemi field theory does, however, have much in common with the global workspace theory (GWT) proposed by Bernard Baars [55-58] and others [59]. GWT proposes that the contents of consciousness are information distributed in different parts of the brain that are nonetheless made available globally for diverse cognitive processes including attention, evaluation, memory and verbal reports. However, GWT is a theoretical construct that does not propose a physical site for the global workspace, a physical mechanism for its generation, nor a physical mechanism by which it generates conscious outputs. In contrast, the cemi field theory proposes a physical location for the mind's global workspace: the brain's EM field. The cemi field theory can thereby be considered to be a global workspace theory. Furthermore, unlike traditional GWT, the cemi field theory proposes a physical mechanism for generating the global workspace: neural firing. Last, and once again in contrast to GWT, the cemi field theory proposes a physical mechanism whereby the contents of the (brain EM field) global workspace generates motor outputs through the influence of the brain's EM field on neural firing that lead to motor actions. I now discuss experimental data supporting this last premise.

\section{Evidence of a Role for Endogenous EM Fields Influencing Neuronal Dynamics in the Brain}

Although there was considerable controversy at the turn of the 19th/20th century as to mechanism of communication between neurons, by the middle of the 20th century, the consensus was that neurons communicate primarily by chemical synapses. A strong prediction of the cemi field theory is that neurons also communicate through EM field interactions. It was established, even in the 19th century, that neurons also communicate non-synaptically [60]. For example, the pioneering neuroscientist, du Bois-Reymond, demonstrated that the firing of a nerve may induce the firing of an adjacent nerve. Nonsynaptic communication between neurons [60] may involve diffusion of paracrine effectors such as nitric oxide or carbon monoxide as well as diffusion of ions along electrical gradients, all of which can modulate neuronal sensitivity. Of interest to this article is non-synaptic communication via electric fields, which tends to be divided into ephaptic, which covers short-range interactions and involve small numbers of neurons as in du Bois-Reymond's pioneering experiments, or field effects that have relatively long range and involve large numbers of neurons. However, ephaptic and field transmission terms are used interchangeably in the literature and there is no sharp boundary between them. Ephaptic transmission has been implicated in several pathological conditions such as tinnitus [61] and epileptic seizures [62], but its role in normal brain function remains controversial.

Detecting the role of field-based signals on neuronal dynamics is challenging. The standard approach to investigate function of a biological component is to remove it by, for example, gene knockout or inhibitor, and investigate the phenotypic consequence. This is not possible for EM fields since as they are intrinsic to the motion of charges that are fundamental to neuronal excitation: you cannot have one without the other. Therefore, although it is routine to, for example, investigate the role of a neurotransmitter by adding an antagonist drug or toxin, there are no EM field antagonists. Researchers have therefore relied instead on indirect approaches that either demonstrate non-synaptic transmission of signal between distant neurons or application of external EM fields to nervous tissue to simulate the influence of endogenous fields.

There is now abundant evidence that external EM fields can influence brain function. In transcranial magnetic stimulation (TMS), a current passing through a coil placed on the scalp of subjects generates a time-varying magnetic field that penetrates the skull. TMS has been shown to have a variety of cognitive [63] and therapeutic [64] effects. Its mechanism remains uncertain. TMS-induced magnetic fields are much larger than endogenous magnetic fields so are not considered physiologically relevant. TMS-induced electric 
fields (orthogonal to the magnetic field induced by coils) cannot be measured directly but, from modelling studies, are estimated to be in the range of 50 to $130 \mathrm{mV} / \mathrm{mm}$ [65] and 20-150 mV/mm [66], which are in the range of local field potentials (LFPs) measured with external electrodes (see below) so may be the driver of TMS effects.

Direct evidence for external electric fields influencing neuronal dynamics has been obtained from several experimental systems. Yuji Ikegaya's laboratory in Tokyo found that rat hippocampal brain slice preparations exhibited no spontaneous gamma oscillatory activity. However, gamma oscillations could be reliably induced by application of the muscarinic agonist carbachol and detected using extracellular electrodes [67]. To examine whether the electric fields generated by these rhythmic oscillations affected neural firing patterns in the tissue, they applied an oscillating $40 \mathrm{~Hz}$ electric field that generated an extracellular field in the tissue with an amplitude and waveform similar to those of the carbachol-induced activity. They then stimulated a CA3 pyramidal cell (by injection of current) and measured the delay (latency) in the neuron's repose: a spike. In the absence of external electric field, the spike latency was $\sim 38 \mathrm{~ms}$, but in the presence of an external oscillating field the spike was delayed to $\sim 160 \mathrm{~ms}$ and then followed by a more sustained rhythmic firing burst. When the imposed field was terminated the spiking pattern returned to the control levels. Clearly the external fields were modulating the firing pattern of individual neurons. The researchers also observed that the phase difference between the stimulus and applied field significantly affected the latency of the response. The researchers concluded that their experiments demonstrated the existence of 'a novel mode of interneuronal communication mediated by local electric field'.

David McCormick's laboratory at Yale [68] used multisite depth electrodes to record local field potentials (LFPs) in the primary visual cortex of anaesthetized ferrets. They detected slow oscillations in the endogenous electric field (EF) with peak strength of $\sim 2$ $\mathrm{mV} / \mathrm{mm}$. To examine whether these fields are capable of modulating neural activity in brain tissue, they applied external sine wave fields with the approximate strength of the weak in vivo $\mathrm{EF}^{\prime} \mathrm{s}$ to in vitro brain slice preparations of the ferret visual cortex that spontaneously generate a slow oscillation. Application of the external field caused small membrane depolarizations ( $1 \mathrm{mV}$ drop in the transmembrane voltage) in individual neurones and accelerated neocortical slow oscillations in the in vitro slices which made them more periodic, effectively entraining the slow oscillation. They also applied a naturalistic waveform to the slices and demonstrated that this was also capable of strongly modulating the spontaneous oscillatory activity of the brain tissue; EFs as weak as $0.25-0.5 \mathrm{mV} / \mathrm{mm}$ were able to modify network behaviour. These results led the researchers to propose that the endogenous EFs provide a positive feedback loop that entrains oscillatory networks. To test this hypothesis, they calculated the fields generated by endogenous oscillations in the slices and then applied external fields that either positively or negatively interfered with the predicted endogenous fields. As expected, the externally-enhanced positive feedback promoted oscillatory activity whereas the externally applied negative feedback suppressed the same activity. The researchers concluded that their studies "support a functional role of the endogenous field in guiding physiological network activity by feedback interactions in neocortex".

In another study conducted by Christof Koch's laboratory at the California Institute of Technology [69], both extracellular and intracellular electric fields were monitored by placing multiple electrodes both within and between pyramidal neurones in rat brain slices maintained in vitro. Using one of the electrodes as a source, the researchers were able to apply a weak external (to the neurones) EF (of $\sim 1 \mathrm{mV} / \mathrm{mm}$ ) and (while simultaneously blocking synaptic transmission) record both extracellular and intracellular EF changes in nearby neurones. Application of weak oscillating external fields (of similar magnitude and frequency as endogenous fields) caused measurable changes in the intracellular fields and extracellular EFs and thereby shifted the transmembrane potential of adjacent neurones by $\sim 0.5 \mathrm{mV}$. These transmembrane potential perturbations oscillated at the same frequency as the externally applied fields. To investigate whether these fields have functional signifi- 
cance, the experimenters injected a constant current into the cell body of 25 target neurones to induce (2-4 Hz) spiking and examined the effect of applying an extracellular field on their firing. The applied field did not change the frequency of firing, but it did affect their phase, causing them to preferentially fire at a preferred phase relative to the external field oscillations (in synchrony). Increasing the field strength enhanced the phase locking of the spikes to the applied field. The researchers concluded that, "Endogenous brain activity can causally affect neural function through field effects under physiological conditions", and that the resulting synchronization "may have a substantial effect on neural information processing and plasticity".

These pioneering papers were followed by several additional studies $[62,69-74]$ that extended the demonstration of ephaptic/field interactions into several other systems. One of the most impressive is a recent study from Wade G. Regehr's laboratory at Harvard Medical School who used a combination of in vivo multielectrode recordings to measure both intracellular and extracellular voltages with dynamic clamping and optogenetics in live rodents and rodent brain slices [72]. The team studied the interaction between climbing fibres originating from inferior olive that make excitatory synapses onto Purkinje cells dendrites in the cerebellar cortex. This interaction plays a central role in cerebellar learning and controls the output of the cerebellar cortex into motor actions. The PC can fire in two distinctive ways: a simple spike of frequency around $100 \mathrm{~Hz}$ and a complex spike at $\sim 2 \mathrm{~Hz}$. The team demonstrated that excitatory inputs from climbing fibres into Purkinje cells generated complex spikes associated with large negative extracellular potentials that suppressed simple spikes in neighbouring Purkinje cells through ephaptic coupling. In this way, a single climbing fibre originating from the inferior olive was found to suppress the firing of around 100 Purkinje cells through ephaptic coupling. In turn, suppression of Purkinje cells was shown to transiently disinhibit and thereby promote firing of neurons in the deep cerebellar nuclei involved in sensorimotor control of voluntary motions: willed actions.

In their recent review of ephaptic coupling to the brain's endogenous EM fields [69], the neurophysiologists provocatively ask, but do not answer, the question "why bother?" Why, when neurons have evolved precise wire-like neuronal synaptic communication, should they also accept the kind of inputs that electrical engineers term electrical interference and take great pains to engineer out of their circuits? The first point is that it is clearly a very ancient mode of neuronal communication as it is found not only in vertebrates but also in insects [75], arthropods [76] and molluscs [77]. EM field communication between neurons has been thereby subject to natural selection and evolution for hundreds of millions of years. It seems likely that EM field interactions provide both advantages and disadvantages in neuronal communication depending on the role and architecture of different neuronal networks. Advantages could include fast signalling, phase-locking of multiple downstream EM field-sensitive neurons to the same stimulus, or rapid distribution of EM-field encoded information to many regions of the brain (as in the global workspace model of consciousness). Another advantage might be that it provides the brain with 'field computing' [78] sometimes known as quantum-like computing [79], which has several features in common with quantum computing such as ease of implementation of mathematical functions such as Fourier transforms, compared to digital computers. I have proposed that this form of field computing — algorithms in space rather than in time [29] can only be implemented by neurons interacting through EM fields and is fundamental to consciousness. Negative influences of EM fields on neuronal communication include all the varieties of undesirable "feedback" familiar to both sound and electrical engineers. The action of the brain's EM field on the neuronal computing that generates the brain's EM field, thereby provides both positive and negative self-referencing feedback loops, what the philosopher Douglas Hofstadter terms a "strange loop" [80] that he claimed is central to consciousness.

With positive and negative impacts, natural selection would inevitably have acted to enhance EM field communication when it provided an advantage and inhibit EM field 
communication when it interfered with the function of a neuronal network. Therefore, with the only the assumption that EM/field ephaptic commination is visible to natural selection, the theory of natural selection predicts that the brain would evolve into an EM field-insensitive stream associated with asynchronously firing and non-conscious activities, and an EM field-sensitive stream associated with synchronously firing neurons. This is of course precisely what we find in the human mind and probably in all minds. We call the EM field insensitive stream our non-conscious mind that performs automatic (unwilled) tasks such as coordinating the precise movements of our lips and tongue for making the sound of words. We call the EM field-sensitive stream that outputs voluntary actions driven by our "will", consciousness.

A fascinating parallel to the proposed mechanism by which conscious information in the brain gains access to motor outputs in the cemi field theory is currently being implemented in brain-computer interfaces (BCIs) or brain-machine interfaces (BMIs) that aim to provide paralyzed individuals or those suffering locked-in syndrome with the ability to direct the motion of prosthetic limbs or direct the output of speech synthesisers for communication. The most popular BMI approaches utilize EEG signals-the external manifestation of the brain's endogenous EM field - as the interface between the brain and machine motor outputs [81], effectively implementing the same informational loop from EM field-encoded neuronal information to motor actions as is proposed in the cemi field theory. Further into the future are efforts, such as those by Elon Musk's company Neuralink [82], to enhance human capabilities using neural implant electrode inserted into the brain to the record the brain's extracellular EM field. The, albeit limited at present, success of BMIs proves that information capable of directing consciously willed actions is encoded in the brain's EM field. The cemi field theory merely proposes that, in normally functioning individuals, the brain's endogenous EM field substitutes for EEG with the individual's own body taking the place of the machine. Another strong prediction of the cemi field theory is that subjects using EEG-controlled CMIs and BMI will feel that their machine actions are willed by their conscious mind. Users of the first commercially available CMI (https: / / www.next-mind.com, accessed date 25 August 2021) that uses helmet-mounted EEG sensors placed above the visual cortex to detect to user's centre of focus on a computer screen and thereby control the user's digital environment, report that the system feels "natural" as if they willed the computer-controlled actions.

\section{Predictions of the Cemi Field Theory}

In my earlier cemi field theory papers [13,19,26-29,83], I made several predictions:

(1) Synchronous neuronal firing will correlate with conscious neuronal states.

(2) Stimuli that reach conscious awareness will be associated with EM field modulations that are strong enough to directly influence the firing of neurones that direct motor actions.

(3) Stimuli that do not reach conscious awareness will not be associated with EM field modulations that do not influence the firing of neurones that direct motor actions.

(4) Increased complexity of conscious thinking should therefore correlate with increased complexity of the brain's EM field.

(5) Agents that disrupt the interaction between the brain's EM field and neurones will induce unconsciousness.

(6) Memory will be associated with strong EM field perturbations that alter synaptic connections.

(7) The brain's EM field should be relatively insulated to perturbation from exogenous EM fields encountered in normal environments.

(8) Appropriately-shaped artificial radio frequency or microwave EM fields that penetrate brain tissue should be capable of reinforcing or inhibiting motor response normally associated with event-related potentials.

(9) Arousal and alertness will correlate with conditions in which EM field fluctuations are most likely to influence neurone firing; conversely, low arousal and unconsciousness 
will correlate with conditions when EM fields are least likely to influence neurone firing.

(10) The evolution of consciousness in animals should correlate with an increasing level of electrical coupling between the brain's endogenous EM field and (receiver) neurone firing.

(11) Conventional computers will never be conscious.

(12) Computers that compute through EM field interactions will be conscious.

(13) Consciousness should demonstrate field-level dynamics.

As described here and in my earlier publications, there is abundant evidence for predictions 1-8. Predictions 8-13 remain to be tested.

\section{However, Is the EM Field-Driven Will Free?}

Free will is one of the most notorious problems of philosophy as it appears to fly in the face of one of the most fundamental principles of classical science, determinism, which insists the future state of the universe is solely determined by its past state. Yet, the traditional view, at least in philosophical circles, of the meaning of 'free' term in 'free will' is that, although the inanimate universe may run like a clock, some aspect of our conscious mind, our 'will', is undetermined by the past. Moreover, the notion goes on to insist that this 'free will' is the driver of voluntary actions and therefore has causal efficacy in the world such that some threads of the future state of the cosmos are not determined solely by the past but also by the collective action of those beings, such as humans, that wield free will. We therefore appear to have two sets of rules operating on our universe, one driven by clock-like mechanisms that obey mathematical laws such as Newton's, quantum mechanics or general relativity, and another that obeys no laws but is instead subject to the whims of conscious beings. This dualistic universe, first clearly enunciated by Descartes, is the problem of free will.

The notion of free will has of course also been challenged by the experiments performed by the neurophysiologist, Benjamin Libet in the 1980s, who detected brain activity that predicted a voluntary action many hundreds of milliseconds before the person who willed that action knew that they had actually willed it $[84,85]$. Many philosophers and neuroscientists have since argued that Libet's experiments proved that conscious will is an illusion. Yet Libet himself did not accept that conclusion and instead proposed that his proposed 'conscious mental field' possessed 'a causal ability to affect or alter neuronal function', in effect providing a nudge one way or another towards or away from actions that had been initiated non-consciously.

The cemi field theory takes up Libet's proposal with the brain's EM field providing the nudge [13]. Free will thereby becomes our experience of the influence of the brain's cemi field on neuron firing, as illustrated in Figure 1, what we call free will. This is, of course, a causal influence and fully consistent with determinism.

Moreover, it is interesting to note that the idea of a free will appears to be relatively recent idea. A much more prominent idea in ancient texts and myths is that of fate or destiny which is essentially a deterministic notion in which human lives are controlled, not by their will but by the whims of a God or gods. For example, in Sophocles' play Oedipus the King, the Greek Oedipus myth, King Laius and Queen Jocaster learn from an astrologer that their son, Oedipus will kill his father and marry his mother. To prevent this happening, Oedipus' parents give the child to a shepherd to be left on the hillside where he would be eaten by wolves. However, the shepherd instead gives the child away so he is brought up not knowing his father and so kills him when he meets him at a crossroads. Lauis' attempt to avoid his fate has been the tragic instrument of his fate. Oedipus' marriage to his own mother is similarly tragically inevitable and not affected by any apparent decisions he or she make.

This deterministic notion of destiny and fate unaffected by apparent decisions that we might make is widespread in many traditional cultures. For example, in the African Yoruba culture, 'a Yoruba will say that once destiny is fixed by Oluron it cannot be changed' [86]. It 
is also evident in contemporary Islamic culture in the frequent use of the phrase 'inshallah' or 'as God wills it'. The modern notion of 'free will' instead comes from the later Western Christian tradition that our decisions must be free so that we are culpable for our actions. God rewards the worthy who freely make virtuous decisions with an eternity of heavenly bliss. He condemns the unworthy with an eternity of pain and unhappiness for their freely-made wicked decisions. We have to be free to be good or bad.

The problem of free will is however a pseudo problem generated by generations of theologians and philosophers who have accepted this non-deterministic notion of free will, despite the fact that it is inconsistent with our subjective experience of the action of our will. Our subjective experience is not of making decisions that are free in the sense of having no preceding cause. If we examine our thoughts when we initiate an action driven by our free will, such as picking up the telephone, and examine the thoughts that preceded that action, then we do not usually find a blank. Instead, we find that the thoughts that initiated the action were determined by earlier thoughts, such as the memory that we promised to call our friend to arrange a game of tennis. Probing further back, we might recall that we were prompted to think about our friend after spotting an actor in a TV programme who looked a bit like her and so on. Thoughts do not simply pop into heads; they are instead determined by earlier thoughts or events that are the cause of our present thoughts. It is not that free will is an illusion that is denied by determinism, it is just that philosophers have been arguing about a phenomenon that does not correspond to our actual subjective experience, which is of a causal, rather than 'free' will.

The cemi field theory proposes that the will is our experience of the interaction between the brain's EM field and neurons that leads to motor actions, such as speech. As reviewed in this article, there is abundant evidence that ephaptic/field effects modify neural firing in circuits that are involved in voluntary motor actions, such as in the cerebellar cortex. The influence may sometimes prompt neurons to fire or, at other times, inhibit neurons from firing. Curiously, this kind of influence is identical to the role proposed for consciousness by William James more than a century ago when he suggested that the cortex is delicately balanced with a 'hair-trigger' such that the slightest jar or accident could set it firing erratically, yet 'if consciousness can load the dice, can exert a constant pressure in the right direction, can feel what nerve processes are leading to the goal, can reinforce and strengthen these and at the same time inhibit those that threaten to lead astray, why, consciousness will be of invaluable service' [87].

The cemi field theory proposes that the brain's EM field is what 'can load the dice, can exert a constant pressure in the right direction'. This is what we experience as the will. It is causal, but it differs from our experience of non-conscious involuntary actions because our conscious mind-the brain's EM field-is in the driving seat exerting the pressure that leads to actions. That pressure is driven by the most complex and dynamic EM object in the known universe, the cemi field, to deliver actions that are better informed than those driven solely by neuronal wires, providing James' 'invaluable service'. Will is not an illusion. It is our experience of EM field-level control. It may be the greatest gift that evolution has conferred on humanity.

\section{Discussion}

The cemi field theory provides a physical theory of consciousness that, as far as I am aware, is consistent will all known facts of conscious experience. Unlike other popular theories of consciousness, such as Integrated Information Theory or Global Workspace theory, the cemi field theory provides a physical rather than merely a theoretical description of consciousness. It envisages consciousness as EM field information encoded in the brains' EM field experienced by the brain's EM field. Again, unlike most other theories of consciousness, the theory provides many testable predictions, most of which have been validated by experimental findings. By shifting the substrate of consciousness from the particulate matter of the brain to the equally physical but immaterial EM field of the brain, the cemi field theory effortlessly solves the binding problem. It also, for the first time, 
provides a clear physical distinction between conscious and non-conscious neural activity as well as between non-conscious and willed actions. It envisages what we call 'free will' as our experience of the influence of the brain's EM on neural firing patterns that lead to motor actions.

Of course, like all theories of consciousness, more theoretical, experiment and philosophical research is needed to thoroughly explore and test the cemi field theory. On the theoretical side, more work needs to be done on the mathematical structure of the theory in order to deliver accurate predictions capable of being verified/refuted. On the experimental side, a great deal more could be investigated on the interaction between brain tissue and EM fields. AI may also be a promising avenue for future experimental research, particularly if computers could be designed that compute through EM field interactions as well as neuronal/synaptic connections. On the philosophical side, a good deal of work needs to be done to gain an understanding of what it might feel like to be the brain's endogenous EM field. For example, how would an EM field that travels at the speed of light, and thereby experiences zero time between transmitter and receiver, experience the arrow of time? Understanding the implications of the cemi field theory is challenging, but it could provide the key to unlocking one of the last great mysteries of science.

Funding: This research received no external funding.

Institutional Review Board Statement: Not applicable.

Informed Consent Statement: Not Aplicable.

Data Availability Statement: Not applicable.

Conflicts of Interest: The author declares no conflict of interest.

\section{References}

1. Köhler, W. Dynamics in Psychology; WW Norton \& Company: New York, NY, USA, 1960.

2. Popper, K.R.; Lindahl, B.I.; Arhem, P. A discussion of the mind-brain problem. Theor. Med. 1993, 14, 167-180. [CrossRef]

3. Lindahl, B.I.; Arhem, P. Mind as a force field: Comments on a new interactionistic hypothesis. J. Theor. Biol. 1994, 171, 111-122. [CrossRef] [PubMed]

4. Libet, B. A testable field theory of mind-brain interaction. J. Conscious. Stud. 1994, 1, 119-126.

5. Libet, B. Conscious mind as a field. J. Theor. Biol. 1996, 178, 223-226. [CrossRef] [PubMed]

6. Hameroff, S.; Penrose, R. Orchestrated reduction of quantum coherence in brain microtubules: A model for consciousness. Math. Comput. Simul. 1996, 40, 453-480. [CrossRef]

7. Penrose, R. The Emperor's New Mind; Oxford University Press: London, UK, 1989.

8. Penrose, R. Shadows of the Mind; Oxford University Press: London, UK, 1995.

9. McFadden, J.; Al-Khalili, J. Life on the Edge: The Coming of Age of Quantum Biology; Crown Publishing Group: New York, NY, USA, 2016.

10. Cobb, M. The Idea of the Brain: The Past and Future of Neuroscience; Hachette: London, UK, 2020.

11. Crick, F. The Astonishing Hypothesis; Simon and Schuster: New York, NY, USA, 1994.

12. Crick, F.; Koch, C. The problem of consciousness. Sci. Am. 1992, 267, 152-159. [CrossRef] [PubMed]

13. McFadden, J. Synchronous firing and its influence on the brain's electromagnetic field: Evidence for an electromagnetic theory of consciousness. J. Conscious. Stud. 2002, 9, 23-50.

14. Kreiter, A.K.; Singer, W. Stimulus-dependent synchronization of neuronal responses in the visual cortex of the awake macaque monkey. J. Neurosci. 1996, 16, 2381-2396. [CrossRef] [PubMed]

15. Wilke, M.; Logothetis, N.K.; Leopold, D.A. Local field potential reflects perceptual suppression in monkey visual cortex. Proc. Natl. Acad. Sci. USA 2006, 103, 17507-17512. [CrossRef]

16. Lutz, A.; Lachaux, J.-P.; Martinerie, J.; Varela, F.J. Guiding the study of brain dynamics by using first-person data: Synchrony patterns correlate with ongoing conscious states during a simple visual task. Proc. Natl. Acad. Sci. USA 2002, 99, 1586-1591. [CrossRef]

17. Steinmann, S.; Leicht, G.; Ertl, M.; Andreou, C.; Polomac, N.; Westerhausen, R.; Friederici, A.D.; Mulert, C. Conscious auditory perception related to long-range synchrony of gamma oscillations. Neuroimage 2014, 100, 435-443. [CrossRef] [PubMed]

18. McFadden, J. Quantum Evolution; HarperCollins: London, UK, 2000.

19. McFadden, J.J. The Conscious Electromagnetic Information (Cemi) Field Theory:The Hard Problem Made Easy? J. Conscious. Stud. 2002, 9, 45-60.

20. Pockett, S. The Nature of Consciousness: A. Hypothesis; Writers Club Press: Lincoln, NE, USA, 2000. 
21. Pockett, S. Difficulties with the electromagnetic field theory of consciousness. J. Conscious. Stud. 2002, 9, 51-56. [CrossRef]

22. John, E.R. A field theory of consciousness. Conscious. Cogn. 2001, 10, 184-213. [CrossRef]

23. John, E.R. The neurophysics of consciousness. Brain Res. Rev. 2002, 39, 1-28. [CrossRef]

24. Fingelkurts, A.A.; Fingelkurts, A.A. Brain-mind operational architectonics imaging: Technical and methodological aspects. Open Neuroimaging J. 2008, 2, 73-93. [CrossRef] [PubMed]

25. Fingelkurts, A.A.; Fingelkurts, A.A.; Neves, C.F. Natural world physical, brain operational, and mind phenomenal space-time. Phys. Life Rev. 2001, 7, 195-249. [CrossRef]

26. McFadden, J. The CEMI Field Theory: Seven Clues to the Nature of Consciousness. In The Emerging Physics of Consciousness; Tuszynski, J., Ed.; Springer: New York, NY, USA, 2006; pp. 387-406.

27. McFadden, J. The CEMI Field Theory Gestalt Information and the Meaning of Meaning. J. Conscious. Stud. 2013, 20, 152-182.

28. McFadden, J. The CEMI field theory closing the loop. J. Conscious. Stud. 2013, 20, 153-168.

29. McFadden, J. Integrating information in the brain's EM field: The cemi field theory of consciousness. Neurosci. Conscious. 2020, 2020, niaa016. [CrossRef]

30. Edelman, G.M.; Tononi, G. Consciousness: How Matter Becomes Imagination; Penguin: London, UK, 2013.

31. Koch, C.; Massimini, M.; Boly, M.; Tononi, G. Neural correlates of consciousness: Progress and problems. Nat. Rev. Neurosci. 2016, 17, 307. [CrossRef] [PubMed]

32. Srinivasan, R.; Russell, D.P.; Edelman, G.M.; Tononi, G. Increased synchronization of neuromagnetic responses during conscious perception. J. Neurosci. 1999, 19, 5435-5448. [CrossRef] [PubMed]

33. Tononi, G. An information integration theory of consciousness. BMC Neurosci. 2004, 5, 42. [CrossRef] [PubMed]

34. Tononi, G. Consciousness as integrated information: A provisional manifesto. Biol. Bull. 2008, 215, 216-242. [CrossRef] [PubMed]

35. Tononi, G.; Edelman, G.M. Consciousness and complexity. Science 1998, 282, 1846-1851. [CrossRef]

36. Tononi, G.; Koch, C. The neural correlates of consciousness. Ann. N. Y. Acad. Sci. 2008, 1124, 239-261. [CrossRef]

37. Tononi, G.; Boly, M.; Massimini, M.; Koch, C. Integrated information theory: From consciousness to its physical substrate. Nat. Rev. Neurosci. 2016, 17, 450. [CrossRef]

38. Searle, J. Can Information Theory Explain Consciousness. Available online: https://www.nybooks.com/articles/2013/01/10 / can-information-theory-explain-consciousness / (accessed on 27 August 2021).

39. Cerullo, M.A. The problem with phi: A critique of integrated information theory. PLoS Comput. Biol. 2015, 11, e1004286. [CrossRef]

40. Matsuo, Y.; Mori, J.; Hamasaki, M.; Nishimura, T.; Takeda, H.; Hasida, K.; Ishizuka, M. Polyphonet: An advanced social network extraction system from the web. J. Web Semant. 2007, 5, 262-278. [CrossRef]

41. Spertus, E.; Sahami, M.; Buyukkokten, O. Evaluating similarity measures: A large-scale study in the orkut social network. In Proceedings of the Eleventh ACM SIGKDD International Conference on Knowledge Discovery in Data Mining; ACM: New York, NY, USA, 2005; pp. 678-684.

42. McDaid, A.F.; Greene, D.; Hurley, N. Normalized mutual information to evaluate overlapping community finding algorithms. arXiv 2011, arXiv:11102515.

43. Ahn, Y.-Y.; Bagrow, J.P.; Lehmann, S. Link communities reveal multiscale complexity in networks. Nature 2010, 466, 761. [CrossRef]

44. Hirata, H.; Ulanowicz, R.E. Information theoretical analysis of ecological networks. Int. J. Syst. Sci. 1984, 15, 261-270. [CrossRef]

45. Fiedor, P. Networks in financial markets based on the mutual information rate. Phys. Rev. E 2014, 89, 052801. [CrossRef] [PubMed]

46. Leydesdorff, L. The mutual information of university-industry-government relations: An indicator of the Triple Helix dynamics. Scientometrics 2003, 58, 445-467. [CrossRef]

47. Pompe, B.; Blidh, P.; Hoyer, D.; Eiselt, M. Using mutual information to measure coupling in the cardiorespiratory system. IEEE Eng. Med. Biol. Mag. 1998, 17, 32-39. [CrossRef] [PubMed]

48. Meyer, P.E.; Lafitte, F.; Bontempi, G. Minet: AR/Bioconductor package for inferring large transcriptional networks using mutual information. BMC Bioinform. 2008, 9, 461. [CrossRef] [PubMed]

49. Amiri, F.; Yousefi, M.R.; Lucas, C.; Shakery, A.; Yazdani, N. Mutual information-based feature selection for intrusion detection systems. J. Netw. Comput. Appl. 2011, 34, 1184-1199. [CrossRef]

50. Bowsher, C.G. Information processing by biochemical networks: A dynamic approach. J. R. Soc. Interface 2010, 8, 186-200. [CrossRef]

51. Bourgault, F.; Makarenko, A.A.; Williams, S.B.; Grocholsky, B.; Durrant-Whyte, H.F. Information based adaptive robotic exploration. In Proceedings of the IEEE/RSJ International Conference on Intelligent Robots and Systems, Lausanne, Switzerland, 30 September-4 October 2002; pp. 540-545.

52. Gabrié, M.; Manoel, A.; Luneau, C.; Macris, N.; Krzakala, F.; Zdeborová, L. Entropy and mutual information in models of deep neural networks. J. Stat. Mech. Theory Exp. 2019, 2019, 124014. [CrossRef]

53. Ribeiro, A.S.; Kauffman, S.A.; Lloyd-Price, J.; Samuelsson, B.; Socolar, J.E. Mutual information in random Boolean models of regulatory networks. Phys. Rev. E 2008, 77, 011901. [CrossRef]

54. Kakigi, R.; Koyama, S.; Hoshiyama, M.; Kitamura, Y.; Shimojo, M.; Watanabe, S. Pain-related magnetic fields following painful CO2 laser stimulation in man. Neurosci. Lett. 1995, 192, 45-48. [CrossRef]

55. Baars, B.J. A Cognitive Theory of Consciousness; Cambridge University Press: New York, NY, USA, 1988. 
56. Baars, B.J. How does a serial, integrated and very limited stream of consciousness emerge from a nervous system that is mostly unconscious, distributed, parallel and of enormous capacity? In Experimental and Theoretical Studies of Consciousness; Wiley: Chichester, UK, 1993; pp. 282-303.

57. Baars, B.J. Global workspace theory of consciousness: Toward a cognitive neuroscience of human experience. Prog. Brain Res. 2005, 150, 45-53.

58. Baars, B.J.; Gage, N.M. Cognition, Brain, and Consciousness: Introduction to Cognitive Neuroscience; Academic Press: Cambridge, MA, USA, 2010.

59. Deco, G.; Vidaurre, D.; Kringelbach, M.L. Revisiting the global workspace orchestrating the hierarchical organization of the human brain. Nat. Hum. Behav. 2021, 5, 497-511. [CrossRef]

60. Jefferys, J. Nonsynaptic modulation of neuronal activity in the brain: Electric currents and extracellular ions. Physiol. Rev. 1995, 75, 689-723. [CrossRef] [PubMed]

61. Eggermont, J.J. On the pathophysiology of tinnitus; a review and a peripheral model. Hear. Res. 1990, 48, 111-123. [CrossRef]

62. Shivacharan, R.S.; Chiang, C.C.; Wei, X.; Subramanian, M.; Couturier, N.H.; Pakalapati, N.; Durand, D.M. Neural recruitment by ephaptic coupling in epilepsy. Epilepsia 2021, 62, 1505-1517. [CrossRef]

63. Pascual-Leone, A.; Walsh, V.; Rothwell, J. Transcranial magnetic stimulation in cognitive neuroscience-virtual lesion, chronometry, and functional connectivity. Curr. Opin. Neurobiol. 2000, 10, 232-237. [CrossRef]

64. Wassermann, E.M.; Zimmermann, T. Transcranial magnetic brain stimulation: Therapeutic promises and scientific gaps. Pharmacol. Ther. 2012, 133, 98-107. [CrossRef]

65. Epstein, A.M. The outcomes movement-Will it get us where we want to go? N. Engl. J. Med. 1990, 323, 266-270. [CrossRef]

66. Ruohonen, J.; Ollikainen, M.; Nikouline, V.; Virtanen, J.; Ilmoniemi, R.J. Coil design for real and sham transcranial magnetic stimulation. IEEE Trans. Biomed. Eng. 2000, 47, 145-148. [CrossRef] [PubMed]

67. Fujisawa, S.; Matsuki, N.; Ikegaya, Y. Chronometric readout from a memory trace: Gamma-frequency field stimulation recruits timed recurrent activity in the rat CA3 network. J. Physiol. 2004, 561, 123-131. [CrossRef]

68. Frohlich, F.; McCormick, D.A. Endogenous electric fields may guide neocortical network activity. Neuron 2010, 67, 129-143. [CrossRef]

69. Anastassiou, C.A.; Koch, C. Ephaptic coupling to endogenous electric field activity: Why bother? Curr. Opin. Neurobiol. 2015, 31, 95-103. [CrossRef] [PubMed]

70. Anastassiou, C.A.; Perin, R.; Markram, H.; Koch, C. Ephaptic coupling of cortical neurons. Nat. Neurosci. 2011, 14, 217-223. [CrossRef]

71. Binczak, S.; Eilbeck, J.; Scott, A.C. Ephaptic coupling of myelinated nerve fibers. Phys. D Nonlinear Phenom. 2001, 148, 159-174. [CrossRef]

72. Han, K.-S.; Guo, C.; Chen, C.H.; Witter, L.; Osorno, T.; Regehr, W.G. Ephaptic coupling promotes synchronous firing of cerebellar purkinje cells. Neuron 2018, 100, 564-578. [CrossRef]

73. Martinez-Banaclocha, M. Ephaptic coupling of cortical neurons: Possible contribution of astroglial magnetic fields? Neuroscience 2018, 370, 37-45. [CrossRef]

74. Ruffini, G.; Salvador, R.; Tadayon, E.; Sanchez-Todo, R.; Pascual-Leone, A.; Santarnecchi, E. Realistic modeling of mesoscopic ephaptic coupling in the human brain. PLoS Comput. Biol. 2020, 16, e1007923. [CrossRef] [PubMed]

75. Su, C.-Y.; Menuz, K.; Reisert, J.; Carlson, J.R. Non-synaptic inhibition between grouped neurons in an olfactory circuit. Nature 2012, 492, 66-71. [CrossRef]

76. Berdan, R.C. Intercellular communication in arthropods. In Cell-to-Cell Communication; Springer: Boston, MA, USA, 1987; pp. 299-370.

77. Ramon, F.; Moore, J.W. Ephaptic transmission in squid giant axons. Am. J. Physiol. Cell Physiol. 1978, 234, C162-C169. [CrossRef]

78. MacLennan, B.J. Field computation in natural and artificial intelligence. Inf. Sci. 1999, 119, 73-89. [CrossRef]

79. Khrennikov, A. Quantum-like model of processing of information in the brain based on classical electromagnetic field. Biosystems 2011, 105, 250-262. [CrossRef] [PubMed]

80. Hofstadter, D.R. I Am a Strange Loop; Basic Books: New York, NY, USA, 2007.

81. Mahmood, M.; Kwon, S.; Kim, Y.-S.; Siriaraya, P.; Choi, J.; Boris, O.; Kang, K.; Jun Yu, K.; Jang, Y.C.; Ang, C.S.; et al. Wireless Soft Scalp Electronics and Virtual Reality System for Motor Imagery-based Brain-Machine Interfaces. Adv. Sci. 2021. [CrossRef] [PubMed]

82. Musk, E. An integrated brain-machine interface platform with thousands of channels. J. Med. Internet Res. 2019, 21, e16194. [CrossRef] [PubMed]

83. McFadden, J. Conscious Electromagnetic (CEMI) Field Theory. Neuroquantology 2007, 5, 262-270. [CrossRef]

84. Libet, B.; Gleason, C.A.; Wright, E.W.; Pearl, D.K. Time of conscious intention to act in relation to onset of cerebral activity (readiness-potential). The unconscious initiation of a freely voluntary act. Brain 1983, 106, 623-642. [CrossRef]

85. Libet, B.; Wright, E.W.J.; Gleason, C.A. Readiness-potentials preceding unrestricted 'spontaneous' vs. pre-planned voluntary acts. Electroencephalogr. Clin. Neurophysiol. 1982, 54, 322-335. [CrossRef]

86. Brown, L.M. (Ed.) African Philosophy: New and Traditional Perspectives; Oxford University Press: London, UK, 2004.

87. James, W. Manuscript Lectures; Harvard University Press: Cambridge, MA, USA, 1988; Volume 19. 